Produto \& Produção, vol. 13 n. 1, p. 114-130 fev. 2012

\title{
Considerações sobre confiabilidade no projeto de submarinos
}

Recebido em 30/10/2011. Aceito em 05/02/2012.

Marcus Sá da Cunha

Escola Politécnica da Universidade de São Paulo

marcus.sa.cunha@gmail.com

\section{Fábio Villas Boas}

Escola Politécnica da Universidade de São Paulo vbfabio@ipt.br

\section{Paulo Carlos Kaminski}

Escola Politécnica da Universidade de São Paulo

pckaminski@usp.br

$O$ projeto de submarinos com $\mathrm{o}$ foco em confiabilidade significa realizar $\mathrm{O}$ desdobramento dos requisitos de confiabilidade para atingir os padrões de desempenho e segurança requeridos. O propósito desse trabalho é estabelecer diretrizes de caráter geral para planejar e desdobrar os requisitos de confiabilidade no projeto de submarinos nucleares. Portanto, foram revistos alguns textos selecionados sobre engenharia da confiabilidade e projeto de submarinos. A motivação para realização desta pesquisa decorre da natureza complexa do submarino nuclear, a sua sofisticação tecnológica e o potencial de acidentes catastrófico. Assim, a questão da confiabilidade é fator crítico de sucesso. São abordados no texto os seguintes itens: O Ciclo de Vida do Submarino; Confiabilidade de Sistemas; O Submarino na Perspectiva de Sistema e Confiabilidade; e Confiabilidade no Projeto de Submarinos. Ressalta-se a necessidade do estabelecimento de um programa formal de confiabilidade na estrutura gerencial do projeto.

Palavras chave: Projeto de Submarinos; Submarinos Nucleares; Engenharia da Confiabilidade.

The submarine project for reliability means the deployment of reliability requirements in order to achieve the performance standards and safety requirements. The purpose of this paper is to establish general guidelines for planning and deploying the reliability requirements in the design of nuclear submarines. Therefore, were revised some selected texts on reliability engineering and design on submarines. The motivation for this research arises from the complex nature of the nuclear submarine, its technological sophistication and the potential for catastrophic accidents. So, reliability is critical to success. In this paper are discussed the following items: The 
Submarine Life Cycle; Systems Reliability; The Submarine in a System Perspective and Reliability, and Reliability in Design Submarine. It is emphasized the need of establishing a formal program of reliability in the management structure of the project.

Keywords: Submarine Project; Nuclear Submarines; Reliability Engineering.

\section{INTRODUÇÃO}

O objetivo do artigo é propor diretrizes, de caráter geral, para o planejamento e desdobramento dos requisitos de confiabilidade no projeto e desenvolvimento de submarinos nucleares.

O Submarino Nuclear é analisado na perspectiva de um sistema complexo, considerando todos os elementos constitutivos do seu ciclo de vida. Isto quer dizer que, na análise do sistema, são considerados assuntos do projeto, construção, comissionamento, operação, suporte, manutenção e descomissionamento.

Submarinos são embarcações submersíveis de emprego militar ou utilizadas em pesquisa ou serviços offshore. Essas últimas podem ser tripuladas ou nãotripuladas. Os submarinos de emprego militar podem ser classificados de acordo com o tipo da planta propulsora: os de propulsão diesel-elétrica ou convencional, e os de propulsão nuclear. Os submarinos nucleares são de dois tipos: 1) balísticos; e 2) de ataque.

O submarino nuclear é um sistema técnico-social caracterizado pela sua complexidade e sofisticação tecnológica. Esses submarinos, por utilizarem energia produzida por reatores nucleares, apresentam potencial de falhas catastróficas. Em geral, o projeto desse tipo de sistema encontra importantes conflitos entre os objetivos de desempenho técnico e a confiabilidade, com obstáculos a algumas opções de design em face de uma abordagem conservadora que privilegia a confiabilidade.

Conceitualmente, a confiabilidade é a capacidade de um item desempenhar uma função requerida, sob condições especificadas, durante um intervalo de tempo. A medida de desempenho de confiabilidade está associada à probabilidade de não ocorrência de falhas em um intervalo de tempo, sob certas condições de operação.

A questão da confiabilidade no projeto de submarinos nucleares apresenta três dimensões: (1) segurança; (2) desempenho técnico; e (3) capacidade de sobrevivência (GUIMARÃES, 1999). A confiabilidade de sistema é influenciada pelas decisões tomadas ao longo dos processos de projeto. As deficiências de projeto podem ter efeitos deletérios no desempenho e segurança do sistema, com perda de vidas humanas, implicando dispêndio significativo de recursos financeiros com correções e alterações do projeto, quando não ocorre a perda total.

No projeto de submarinos procura-se atender aos seguintes requisitos operacionais: velocidade, capacidade de ocultação e capacidade bélica, sem comprometer a confiabilidade da embarcação. Neste caso, a confiabilidade tem importantes implicações na segurança do meio, da tripulação e do ambiente em que opera. A confiabilidade também implica na disponibilidade técnica do submarino para cumprir a sua missão, e confere ao projeto capacidade de resistência para sobreviver a eventos de agressão.

No caso específico do submarino, a sua confiabilidade intrínseca resulta da qualidade do projeto e da sua construção. Entretanto, a confiabilidade ao longo do 
ciclo de operações dependerá também da qualidade do suporte provido pela organização que o opera, como treinamento e qualificação da tripulação, apoio logístico e política de manutenção.

Para a consecução do objetivo do artigo, foi feita uma revisão da literatura sobre projeto de submarinos e engenharia de confiabilidade, com o propósito de identificar as oportunidades de inserir as questões sobre confiabilidade no projeto e desenvolvimento de submarinos.

\section{O CICLO DE VIDA DO SUBMARINO}

O ciclo de vida de um sistema complexo geralmente percorrer os seguintes estágios: projeto, construção, comissionamento, suporte, manutenção e descomissionamento. Guimarães (1999), no caso dos submarinos, apresenta estes estágios de forma mais detalhada: (1) Requisitos de Estado Maior (REM); (2) Requisitos de Alto Nível de Sistemas (RANS); (3) Projeto; (4) Fabricação de componentes; (5) Construção naval; (6) Montagem eletromecânica; (7) Comissionamento; (8) Período de Manutenção; (9) Obras de atualização e modernização; e (10) Descomissionamento.

A decisão de construir um novo submarino, dada a complexidade, os custos, e os riscos envolvidos, resulta de longo e cuidadoso processo de análise estratégica desenvolvido por uma organização. No caso de um submarino militar, a decisão compete ao poder central de um determinado Estado, que identifica as necessidades de prover certas capacidades operacionais à sua Força Naval.

Comparando os processos de desenvolvimento descritos em Kormilitsin e Khalizev (2001) e Burcher e Rydill (1994), os primeiros de origem russa e os segundos, britânica, observa-se que as formas em que organizam as fases de desenvolvimento de submarinos são basicamente as mesmas e similares à apresentada anteriormente. As duas abordagens fortalecem a percepção de que o sucesso do empreendimento depende fundamentalmente da qualidade dos requisitos estabelecidos na fase inicial do projeto. Quanto ao ferramental de projeto aplicado aos sistemas físicos, percebe-se que os autores russos valorizam o uso de métodos empíricos com base em ensaios de modelos em laboratório, enquanto que os britânicos se valem do uso intensivo de métodos computacionais.

O ciclo de vida de um submarino começa com a tradução das necessidades operativas em requisitos de projeto. Etapa decisiva para o sucesso do seu desenvolvimento. Os marcos revisionais ao fim das fases iniciais - planejamento e desenvolvimento de conceitos -, conforme Dieter (2008), é o resultado de análises rigorosas para a tomada de decisão de prosseguimento do projeto para as demais fases. Tal rigor é necessário uma vez que a partir desses pontos haverá aplicação intensiva de recursos, tornando-se altamente onerosa as correções de deficiências de requisitos nas fases mais avançadas. Blanchard e Fabrycky (2006) afirmam que as deficiências dos requisitos iniciais do projeto se refletem ao longo do ciclo de vida do produto, tornando o Custo do Ciclo de Vida demasiadamente alto.

De acordo com as diretrizes vigentes nas Normas para a Logística de Material - EMA-420 (EMA, 2002), as funções básicas de um novo submarino são formalizadas pelos Requisitos de Estado-Maior (REM). Os REM são o resultado de estudos estratégicos balizados por diretrizes de alto nível, onde constam a necessidade geradora, o conceito de emprego e os condicionantes. $\mathrm{Na}$ fase 
seguinte, os REM são desdobrados nos Requisitos de Alto Nível de Sistemas (RANS). Os RANS procuram definir a capacidade operativa de cada sistema do submarino, suas características e requisitos de desempenho, a filosofia de manutenção e apoio logístico pretendido.

A partir dos REM e dos RANS, ainda na fase de concepção, é realizado o Estudo de Exequibilidade (EE), no qual serão propostas e avaliadas as alternativas de projeto viáveis, técnica e economicamente, que solucionem o atendimento aos requisitos pretendidos. Contraposições entre requisitos podem surgir nessa fase, demandando utilização de ferramentas analíticas adequadas em busca da solução ótima global. Caso não seja feita a opção por um projeto consagrado como ponto de partida, a metodologia da espiral de projeto é ferramenta importante para a aproximação e o refinamento do conceito. A espiral de projeto é aplicada por meio de um processo iterativo e harmônico, promovendo ciclos revisionais sucessivos do projeto (KAMINSKI, 2000), conforme exemplificado na figura1.

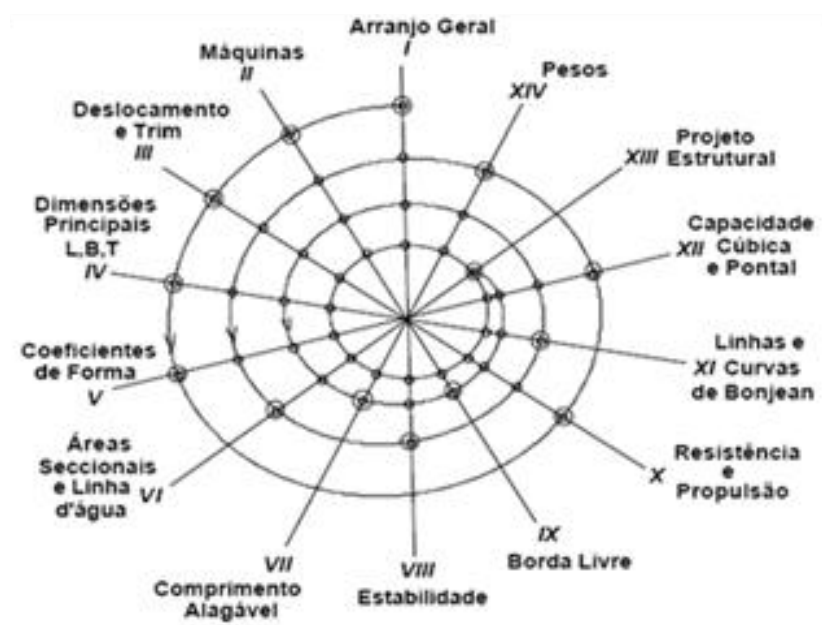

Figura 1 - Espiral de Projeto. Fonte: Vasconcelos e Santos (2010)

O produto final da fase de concepção é o Relatório do Estudo de Exequibilidade, apresentando a consolidação dos RANS e um conjunto de alternativas viáveis. Escolhida a alternativa mais adequada, é então elaborada a edição preliminar das Especificações de Alto Nível de Sistema (EANS) e dá-se o início ao projeto de concepção. O projeto progride ainda pelas seguintes fases: (1) preliminar; (2) contrato; e (3) fase de execução. Ao fim da fase de execução o submarino estará pronto para ser submetido às avaliações operacionais e de engenharia (EMA, 2002).

Dado o longo tempo necessário para o desenvolvimento, a complexidade do produto, os custos envolvidos e a significância dos recursos mobilizados, a construção de um submarino é conduzida de acordo com as melhores práticas de gerenciamento de projeto; as atividades são desempenhadas de forma progressiva por etapas distintas, e são submetidas a mecanismos de controle para garantir o cumprimento dos objetivos finais do projeto.

A obtenção do submarino pode ser feita conduzindo os projetos na forma de Empreendimentos Modulares, estabelecendo uma estrutura gerencial de projeto, explicitando os processos revisionais e decisórios em uma estrutura matricial superposta a uma estrutura organizacional. 


\section{CONFIABILIDADE DE SISTEMAS}

\subsection{Confiabilidade}

A confiabilidade é a "qualidade" de quem ou do que é confiável (HOLANDA, 2004). Ainda no contexto literário, qualidade significa propriedade, atributo, ou condição das coisas (ou das pessoas) capaz de distingui-las das outras e lhes determinar a natureza (HOLANDA, 2004). Na área do conhecimento de administração da produção, "confiabilidade" é um dos cinco objetivos de desempenho operacional (os demais são qualidade, rapidez, flexibilidade e custo), significando "fazer as coisas em tempo" para que os consumidores recebam seus bens e serviços prometidos (SLACK et al, 2008). Assim, é possível depreender que a "confiabilidade" é uma característica de qualidade relacionada ao tempo.

No capítulo em que discorre sobre prevenção e recuperação de falhas, Slack et al (2008) relaciona a confiabilidade com a habilidade de um sistema, produto ou serviço desempenhar, como esperado, uma função, durante certo intervalo de tempo; define também a "disponibilidade" como o tempo útil disponível para operação. A norma IEC 60300-1 (IEC, 2003) apresenta a confiabilidade como um dos elementos da dependabilidade (dependability, no idioma original) e a define com os mesmos termos de Slack et al (2008), devendo ser observadas as condições de uso do sistema. Dependabilidade, de acordo com a referida norma, é o termo empregado para descrever o desempenho em disponibilidade considerando os fatores que a influenciam, de acordo com o representado na figura 2.

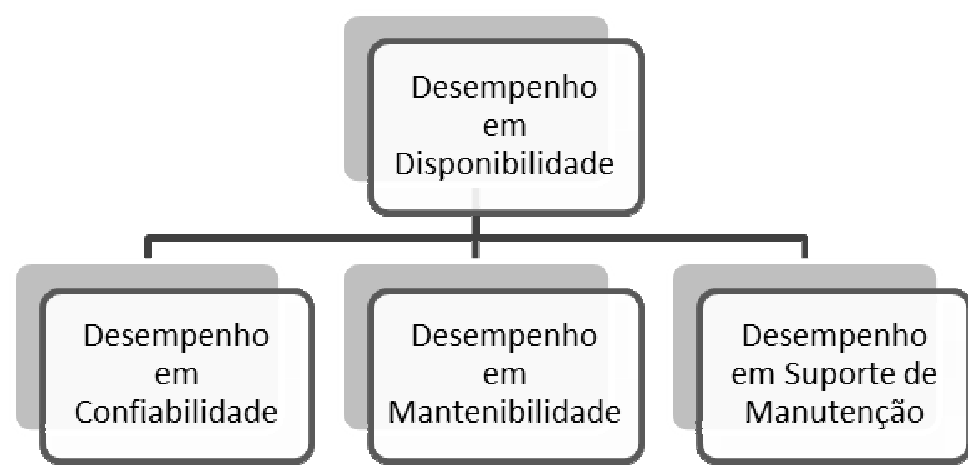

Figura 2 - Elementos da dependabilidade. Fonte: IEC 60300-1 (IEC, 2003)

A norma IEC 60300-1 (IEC, 2003) apresenta as seguintes definições:

(1) Disponibilidade: é a habilidade de um item estar em condições de desempenhar uma função requerida sob certas condições em um dado instante ou ao longo de um dado intervalo de tempo, assumindo que os suprimentos externos necessários estejam providos.

(2) Confiabilidade: é a habilidade de um item desempenhar uma função requerida sob dada condição em um determinado intervalo de tempo.

(3) Mantenabilidade: é a habilidade de um item, sob certa condição de uso, ser mantido ou recolocado em condições de desempenhar uma função requerida, 
quando a sua manutenção é executada sob condições determinadas e mediante procedimentos e meios prescritos.

(4) Suporte de manutenção: é a habilidade de uma "organização de manutenção" prover, sob demanda, os recursos necessários para manter um item em uma condição requerida e de acordo com uma dada política de manutenção.

A dependabilidade é um conceito empregado para descrições genéricas sem a preocupação de quantificá-la. Entretanto os elementos da dependabilidade têm seus respectivos desempenhos avaliados por ferramentas quantitativas baseadas na teoria das probabilidades (O'CONNOR, 2002). Assim, a confiabilidade está relacionada com a probabilidade de não ocorrência de falhas e a mantenabilidade com a probabilidade de um serviço de manutenção ser executado em dado intervalo de tempo (ABNT, 1994). A disponibilidade, também quantificada por probabilidades, pode ser estimada, no regime estacionário com as taxas de falha e de reparo constantes, pela equação (1).

$$
A=\frac{M T B F}{M T B F+M T T R}
$$

A - disponibilidade

MTBF (Mean Time Between Failures) - tempo médio entre falhas: esperança matemática entre falhas de um item.

MTTR (Mean Time to Repair) - tempo de restabelecimento médio: esperança matemática do tempo de restabelecimento.

Além disso, na avaliação da disponibilidade deve ser considerado quantitativamente o desempenho do suporte de manutenção, provendo tratamento estatístico nas avaliações das probabilidades de ocorrência de atrasos nas atividades administrativas e logísticas (ABNT, 1994).

Os avanços e as aplicações em Engenharia da Confiabilidade estão sendo conduzidos pela mudança de paradigma da "economia industrial", onde a centralidade na prestação de serviço está substituindo a ênfase na agregação de valor. Isso quer dizer que a ênfase na agregação de valor ao produto mudou para o valor decorrente do desempenho do produto na provisão do serviço. Assim, a disponibilidade para o serviço passa a ser um dos mais importantes indicadores de desempenho (ZIO, 2009). Reportando-se ao objeto do presente artigo, pode ser inferido que a confiabilidade tem papel relevante na disponibilidade técnica do submarino para desempenhar com eficiência e eficácia a sua missão.

\subsection{Engenharia da Confiabilidade}

A "engenharia da confiabilidade" consolidou-se como engenharia e disciplina científica a partir do emprego sistemático da teoria das probabilidades e de ferramentas estatística para resolver problemas de falhas de componentes eletrônicos (ZIO, 2009). As suas técnicas de avaliação e análise de confiabilidade evoluíram e tiveram seus empregos estendidos a diversos setores, além da eletrônica, sendo seus procedimentos normalizados por diversas entidades internacionais de normas técnicas; O’Connor (2002), Murthy et al (2009) e Zio (2009) referenciam as normas técnicas usuais em engenharia da confiabilidade. 
A ênfase voltada para a confiabilidade no projeto de engenharia não dispensa as melhores práticas de engenharia, de gestão de projetos e da qualidade. Entretanto, a aplicação das melhores práticas dessas áreas de conhecimento não é suficiente para garantir o desempenho em confiabilidade no projeto de sistemas complexos. Portanto, deve ser estabelecido um "Programa de Confiabilidade" formal na estrutura de projeto sempre que os riscos e os custos relacionados com a ocorrência de falhas sejam de magnitudes relevantes (O'CONNOR, 2002).

$\mathrm{O}$ aspecto fundamental na análise de confiabilidade é a incerteza relacionada com ocorrência de falhas e as suas conseqüências (ZIO, 2009). Isto significa fazer considerações em projetos de engenharia sobre as possíveis falhas e a segurança, considerando eventos e cenários de natureza probabilística em ambiente no qual se faz uso intensivo e sistemático de ferramentas determinísticas.

Em suma, a engenharia da confiabilidade é uma atividade científica multidisciplinar bem estabelecida, que fornece um conjunto de métodos formais para investigar os limites da incerteza entre a operação de um sistema e a ocorrência de falhas, abordando as seguintes questões (ZIO, 2009): (1) por que os sistemas falham; (2) como desenvolver sistemas confiáveis; (3) como medir e testar a confiabilidade no projeto, na operação e na gestão; (4) como manter os sistemas confiáveis por meio da manutenção, diagnósticos de falha e prognósticos.

Apesar de todo progresso da engenharia de confiabilidade, alguns temas continuam a desafiar os pesquisadores dessa área de conhecimento (ZIO, 2009): (1) representação e modelagem de sistemas; (2) quantificação dos modelos de sistemas; e (3) representação, propagação e quantificação da incerteza no comportamento dos sistemas. Estes desafios têm se tornado cada vez maiores com o desenvolvimento e operação de sistemas níveis crescentes de complexidade, como é o caso do submarino nuclear.

\subsection{Engenharia de Sistema}

A definição de "sistema" encontrada na norma IEC 60300-1 (IEC, 2003) é simples e abrangente: conjunto de elementos inter-relacionados e iterativos. A referida norma observa que a estrutura de um sistema pode ser hierarquizada e contextualiza a definição de sistema em termos de dependabilidade - um sistema deve ter: (1) um propósito definido, descrito em termos de funções a serem desempenhadas; (2) as condições de operação e uso expressamente estabelecidas; e (3) os limites bem definidos.

A "engenharia de sistemas" é definida como o esforço para (BLANCHARD; FABRYCKY, 2006): (1) traduzir uma necessidade operacional em termos de desempenho e configuração de sistema; (2) otimizar a definição e o projeto do sistema, assegurando a compatibilidade entre os elementos físicos, funcionais e interfaces; e (3) integrar desempenho, produtibilidade, confiabilidade, mantenabilidade, suportabilidade, e outros atributos dentro do esforço global de engenharia.

Nessa visão sistêmica, Zio (2009) considera a disponibilidade como um atributo que depende de uma série de processos inter-relacionados, dentre os quais destaca: (1) degradação dos componentes; (2) falhas e reparos; e (3) diagnóstico e manutenção. Esses processos são resultantes da iteração entre diferentes sistemas incluindo não somente a parte física do sistema, como também as pessoas, os 
sistemas de supervisão e controle baseado em software e a organização de apoio logístico. Assim, a confiabilidade e a conseqüente disponibilidade são consideradas atributos emergentes, uma vez que são resultantes das iterações entre vários elementos de natureza diversa.

\subsection{Projeto com o foco em confiabilidade}

O projeto com foco em confiabilidade tem o propósito de assegurar a confiabilidade do sistema (ou produto) durante o seu projeto e desenvolvimento. De acordo com Das e Pecht (2009) a confiabilidade é assegurada por meio do desempenho de atividades relacionadas com a seleção de materiais e peças, o projeto do produto, a fabricação, a montagem, o transporte e manuseio, a operação, a manutenção e o reparo.

Um programa de confiabilidade deve ser elaborado desde as fases primordiais do projeto, estabelecendo um conjunto de regras para balizar as tomadas de decisão de forma a garantir um nível de confiabilidade adequado ao sistema no ciclo operacional. Murthy et al (2009), para desdobrar os requisitos de confiabilidade no projeto, organizam a seqüência de atividades de projeto em fases com uma estrutura matricial, combinando os níveis de decisão com os estágios de desenvolvimento, de acordo com o ilustrado na figura 3.

Assim, a partir da identificação da necessidade de desenvolver um novo sistema e o detalhamento dos seus atributos em consonância com os objetivos estratégico da organização, atividades previstas na primeira fase, os requisitos de confiabilidade são desdobrados por meio da execução, de forma guiada, de um conjunto de atividades, cuidadosamente elaboradas e posicionadas de acordo com as dimensões nível e estágio da matriz de desenvolvimento.

Estágio 1

(Pré-desenvolvimento)
Estágio 2

(Desenvolvimento)
Estágio 3

(Pós-desenvolvimento)

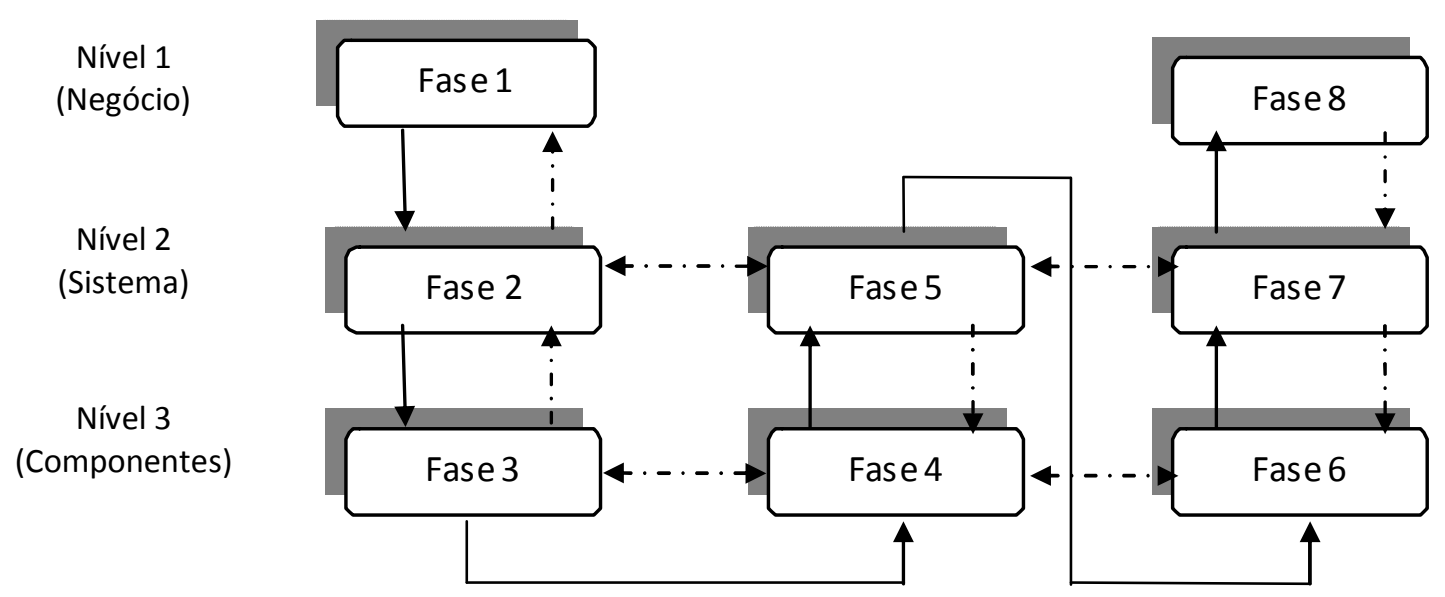

Figura 3 - As fases do ciclo de vida do sistema. Fonte: Murthy et al. (2009) 


\subsection{Apoio logístico integrado}

Apoio Logístico Integrado (ALI) - Integrated Logistics Support (ILS) - é uma ferramenta de gestão integrada do ciclo de vida do sistema que abrange todos os assuntos relacionados ao suporte logístico e operação. O ALI é aplicado por meio de um processo disciplinado e iterativo de planejamento, desenvolvimento e incremento do suporte necessário à operação do sistema (BLANCHARD, 2004).

Por meio do ALI as considerações de suporte logístico têm influência positiva no desenvolvimento de requisitos de projeto, tornando possível definir e integrar os requisitos de suporte durante as fases de projeto e de fabricação, assegurando que estes requisitos estejam da melhor forma possível relacionados ao projeto (e viceversa).

De acordo com o previsto nas normas EMA-420 (EMA, 2002), os assuntos relacionados ao $\mathrm{ALI}$ do futuro submarino começam a ser considerados nos Estudos de Exeqüibilidade. Ainda na fase de concepção da embarcação é constituída uma equipe específica para o desenvolvimento e implantação do ALI. Os requisitos do ALI são desdobrados pelas fases sucessivas do projeto e desenvolvimento até as suas atividades de suporte e manutenção serem absorvidas na estrutura organizacional da Marinha.

\section{O SUBMARINO NA PERSPECTIVA DE SISTEMA E CONFIABILIDADE}

Considerando a sua estrutura analítica, um submarino pode ser entendido como um macro-sistema, formado por dois sistemas principais: a plataforma e o sistema de combate, conforme mostrado na figura 4. O "Sistema Plataforma" é constituído por um conjunto de subsistemas necessários para que a embarcação opere como meio submersível - a plataforma deve ser dotada com capacidade de submergir, emergir, navegar e prover condições de subsistência à vida humana. $\mathrm{O}$ "Sistema de Combate" compreende o conjunto de armas, sensores, comunicação e controle.

De uma forma geral, convencionais ou nucleares, os submarinos apresentam a mesma arquitetura e sistemas básicos: casco com seção reta circular e perfil hidrodinâmico, com uma estrutura no formato de vela na parte superior; propulsores montados na parte de ré da embarcação; superfícies de governo (leme vertical) e de controle de profundidade (lemes horizontais) e tanques de lastro esgotados com ar comprimido de alta pressão. Internamente o submarino possui tanques de compensação e trimagem, necessários para manter o seu equilíbrio hidrostático. $O$ sistema de combate é composto basicamente por tubos lançadores de torpedos ou mísseis, periscópios, sonares e radares. As figuras 5 e 6 mostram as estruturas analíticas básicas da plataforma e do sistema de combate respectivamente. 


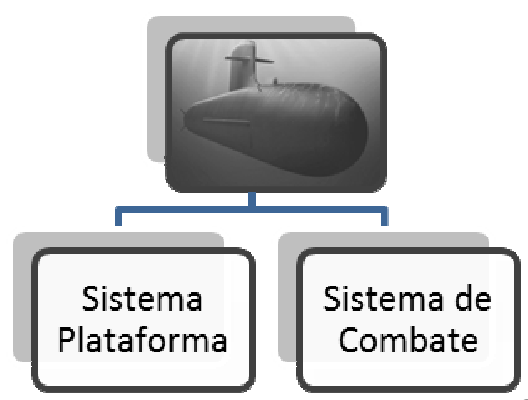

Figura 4 - Estrutura Analítica Macro de um Submarino

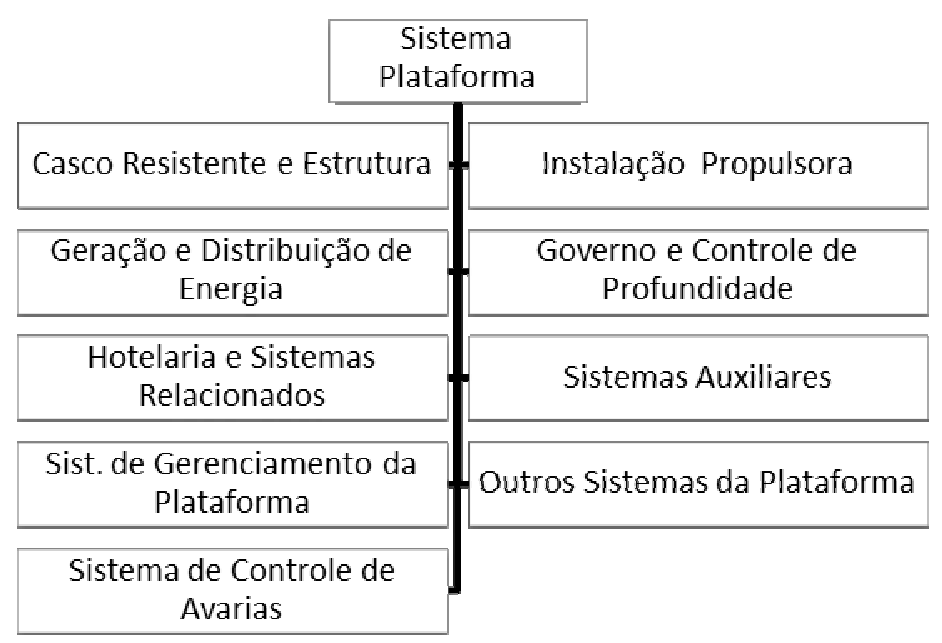

Figura 5 - Estrutura Analítica Básica do Sistema Plataforma

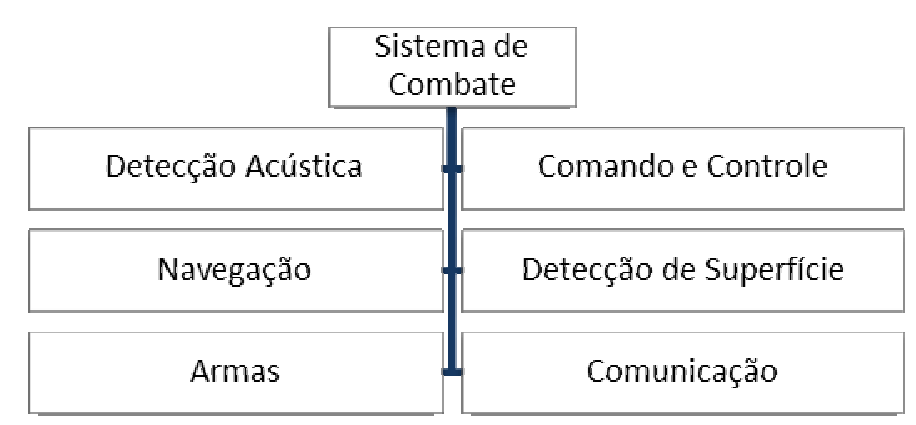

Figura 6 - Estrutura Analítica Básica do Sistema Plataforma

$O$ que diferencia fundamentalmente o submarino convencional do submarino nuclear é o tipo de planta propulsora. A maioria dos submarinos convencionais tem como fonte primária de energia um conjunto de diesel-geradores, que suprem um conjunto de baterias recarregáveis, estas por sua vez fornecem energia aos motores elétricos principais que movimentam o conjunto propulsor (eixos e hélices), e aos sistemas auxiliares. A grande desvantagem deste tipo de propulsão é a sua dependência do ar atmosférico, pois durante o ciclo de carregamento das baterias o submarino emerge, tornando-se vulnerável.

Em menor escala, têm sido empregadas soluções tecnológicas de geração de energia independente do ar atmosférico para as plantas propulsoras de submarinos convencionais, denominadas Air-Independent Propulsion (AIP). Existem duas 
soluções para o emprego de AIP: (1) combustível e oxigênio líquido ou pressurizado; e (2) células de combustível (hidrogênio e oxigênio). O emprego de AIP reduz o problema da vulnerabilidade do submarino convencional, aumentando a sua autonomia, entretanto requer requisitos de segurança mais elevados e torna a logística de suporte mais complexa por empregar elementos químicos de alta periculosidade.

Os submarinos nucleares têm como principal fonte de energia propulsora um reator nuclear. A planta dominante nesse caso é o "Reator de Água Pressurizada", conhecida como Pressurized Water Reactor (PWR). Uma instalação propulsora nuclear baseada em reator PWR consiste em dois circuitos termo-hidráulicos fechados, utilizando água como fluido de trabalho: (1) "Sistema de Resfriamento do Reator" (circuito primário), onde a água é mantida na fase líquida a temperatura e pressão elevadas; (2) "Sistema de Conversão de Energia", onde a água sofre mudança de fase em um ciclo Rankine. Estes circuitos são fisicamente separados, mas acoplados por um trocador de calor do tipo feixe-tubular, denominado "Gerador de Vapor" (GUIMARÃES, 1999).

A confiabilidade no projeto de submarinos nucleares tem três dimensões: desempenho técnico, segurança e capacidade de sobrevivência. O desempenho em confiabilidade, em conjunto com a mantenabilidade, é importante requisito de projeto para assegurar a disponibilidade técnica do submarino a fim de que cumpra as tarefas previstas de forma eficiente e eficaz. Por outro lado, de acordo com as diretrizes de segurança para submarinos nucleares, propostas por Guimarães (1999), a abordagem racional dos problemas decorrentes da aplicação da tecnologia nuclear em plantas propulsoras de submarinos deve englobar:

(1) a proteção da tripulação, do pessoal de apoio logístico em terra e do público geral contra danos à saúde e ao meio ambiente decorrentes da emissão, intencional ou acidental, de substância radioativas ou ionizantes; e

(2) a capacidade de sobrevivência do submarino, não somente aos riscos inerentes à instalação propulsora nuclear, como também os decorrentes das iterações desta planta com os subsistemas da plataforma e do sistema de combate (agressões internas), com o ambiente físico das áreas oceânicas, costeiras e litorâneas em que opera (agressões externas) e com o ambiente tático-operativo naval (agressões de caráter militar).

Em face de sua complexidade e dos riscos de acidentes, Bierly et al (2008) classificam o submarino nuclear como "Organização de Alta Confiabilidade" ou, como apresentada na literatura estrangeira, High-Reliability Organization (HRO). Este conceito é utilizado para fazer referência a sistemas que apresentam as seguintes características: (1) potencial de acidentes catastróficos; (2) alto grau de acoplamento entre os subsistemas; (3) iteratividade complexa entre as partes; e (3) alto nível de responsabilidade e tensão de seus operadores. Assim, esses sistemas lidam ao longo do seu ciclo de vida com o conflito entre a eficiência técnica pretendida e os requisitos de confiabilidade e segurança.

Guimarães (1999) também identifica este importante conflito em relação aos submarinos nucleares, ao dizer que as bases normativas para projeto e operação de sistemas militares navais privilegiam a disponibilidade, como atributo fundamental para assegurar o sucesso de sua missão, enquanto que as referentes às plantas nucleares focam a seguridade.

Bierly et al (2008), para obterem melhor entendimento sobre o papel da inovação e da aprendizagem organizacional no contexto de uma $\mathrm{HRO}$, realizaram um estudo comparativo entre as formas com que americanos e russos 
desenvolveram seus submarinos nucleares de ataque no período de 1970 a 2000 . Os autores elucidam uma postura mais conservadora dos americanos em relação aos russos, ao adotarem estratégia de plataforma e desenho modular, que privilegia a confiabilidade e segurança em detrimento do desempenho. Guimarães (1999), em consonância com a postura conservadora dos projetistas americanos, detectada por Bierly et al (2008), demonstra a necessidade de uma abordagem balanceada entre disponibilidade e seguridade, uma vez que ao se procurar a otimização global do sistema a segurança nuclear não deve comprometer a segurança naval: se a última for comprometida, a primeira fatalmente o será, pois a segurança da instalação nuclear depende em grande parte da segurança do próprio submarino.

A estratégia de plataforma e da modularidade reforça a necessidade de atendimento dos requisitos de confiabilidade e segurança, uma vez que a construção de uma plataforma crível emprega arquitetura e tecnologia consolidadas e confiáveis para desenvolver sistemas. Bierly et al (2008) identificam relações positivas da estratégia de plataforma com a aprendizagem organizacional, e que reforçam a confiabilidade e a segurança das HRO.

De acordo com a seção anterior, Zio (2009) ao analisar os sistemas complexos também inclui na sua estrutura as iterações com o elemento humano, além das iterações com as partes físicas, organizacionais e softwares de supervisão e controle. Uma representação do sistema submarino compatível com o conceito de HRO e adequada à análise de confiabilidade deve considerar todos estes elementos, de acordo com o mostrado na figura 7.

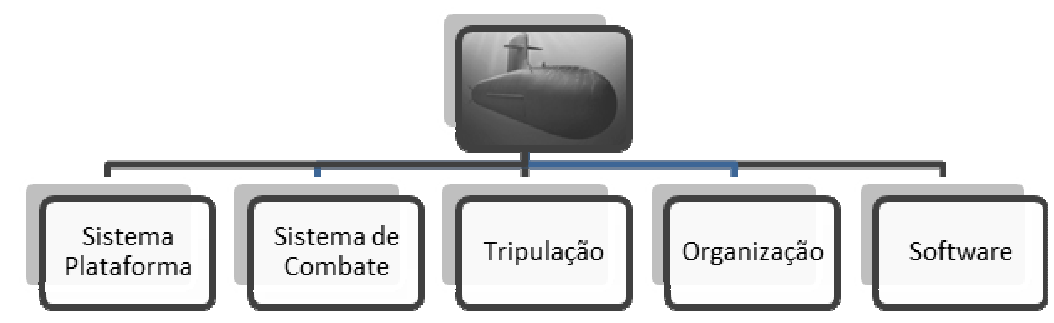

Figura 7 - Estrutura Analítica de um Submarino considerando os elementos humano, organizacional e software

A confiabilidade do sistema submarino resulta das iterações entre os subsistemas representados na figura 7 . Em dado instante de tempo, cada subsistema individualmente pode estar em determinado nível de desempenho, resultante das condições de operação de seus elementos de múltiplos estados, constituindo um sistema de múltiplos estados (ZIO, 2009). A ocorrência de algum estado de desempenho específico de determinado elemento constitutivo de um sistema é um evento probabilístico resultante das condições de operação e das ações de manutenção; a probabilidade de o sistema atingir um determinado nível de desempenho e segurança dependerá da probabilidade de ocorrerem dadas combinações de estados de desempenho de todos os elementos, cada um segundo seu padrão probabilístico. Tal abordagem evidencia a complexidade dos sistemas de múltiplos estados, demandando ferramentas matemáticas e estatísticas sofisticadas para a análise de confiabilidade. 


\section{CONFIABILIDADE NO PROJETO DE SUBMARINOS}

De acordo com que foi analisado nas seções anteriores, diferentemente do projeto de um item de baixa complexidade, o desenvolvimento de um submarino nuclear requer, dentro de sua estrutura organizacional, uma estrutura gerencial para conduzir um programa formal de confiabilidade, tratada no contexto do Apoio Logístico Integrado.

No projeto de submarinos a confiabilidade deve ser tratada como parâmetro de efetividade, devendo ser "explicitamente" especificada e pela qual se deve prover um nível de investimento adequado. Como parâmetro de efetividade, a confiabilidade deve ser analisada em confronto com outros parâmetros de desempenho, buscando otimizar os denominados "trade-off".

Um programa efetivo de confiabilidade fundamenta-se no binômio: responsabilidade e autoridade (O'CONNOR, 2003). Quando a organização reconhece a existência de um projeto, deve ser provido um Gerente do Programa de Confiabilidade. A responsabilidade gerencial deve ser objetivamente definida de forma que um determinado nível de confiabilidade possa ser demonstrado como objetivo alcançável, ou que certos requisitos são suficientes para garantir a não ocorrência de falhas.

O Programa de Confiabilidade deve ser um conjunto de regras para balizar os processos decisórios do projeto que garanta ao submarino o nível de confiabilidade pretendido. Já na fase de concepção devem ser definidos requisitos de projeto relacionados com o desempenho em confiabilidade que apresente o seguinte conteúdo (O'CONNOR, 2002): (1) a definição de falha relacionada com função principal do sistema, cobrindo todos os modos de falhas relevantes para a função; (2) descrição completa e detalhada das características do ambiente operacional, considerando condições armazenamento, transporte, operação e manutenção; (3) enunciados sobre requisitos de confiabilidade ou modos de falhas e efeitos considerados críticos que não devem ocorrer.

A confiabilidade começa a ser desenhada a partir do planejamento do ciclo operativo do submarino. De acordo com a concepção apresentada por Guimarães (1999), a vida útil de 30 anos é organizada em três fases pelas quais se distribuem dois "Ciclos de Atividades Básicos (CAB)", dois "Ciclos de Atividades Intermediários (CAI)", dois "Períodos de Atualização e Modernização (PAM)" e um "Ciclo de Atividade Final (CAF)". A Tabela 1 mostra o perfil planejado de operações com todos os ciclos e as suas respectivas durações em meses.

Tabela 1 - Perfil de Operação de um Submarino Nuclear

\begin{tabular}{clc}
\hline Fase & \multicolumn{1}{c}{ Ciclos e Período } & $\begin{array}{c}\text { Duração } \\
\text { (meses) }\end{array}$ \\
\hline \multirow{3}{*}{ I } & $1^{\circ}$ Ciclo de Atividades Básico (CAB) & 63 \\
& $1^{\circ}$ Ciclo de Atividades Intermediário (CAI) & 57 \\
& Período de Atualização e Modernização (PAM) & 24 \\
& & \\
& & \\
II & $2^{\circ}$ Ciclo de Atividades Básico (CAB) & 63 \\
& $2^{\circ}$ Ciclo de Atividades Intermediário (CAI) & 57 \\
& Período de Atualização e Modernização (PAM) & 24 \\
III & Ciclo de Atividade Final (CAF) & 72
\end{tabular}


Cada Ciclo de Atividades Básico é composto por um "Período Operativo Básico (POB)", com duração de 50 meses, seguido por um "Período de Manutenção Geral (PMG)", de 13 meses. O Período Operativo Básico é dividido 16 "Ciclos Básicos", cada um com duração total de 13 semanas: uma "Missão de Patrulha Básica (MPB)", de nove semanas, seguida de um "Período de Manutenção de Rotina (PMR)" de quatro semanas. A cada oito Ciclos Básicos é previsto um "Período de Docagem de Rotina (PDR)" com duração de quatro meses (GUIMARÃES, 1999). O Ciclo de Atividades Intermediário corresponde a um "Período Operativo Intermediário (POI)", com duração de 57 meses, composto por oito Ciclos Básicos seguido de um Período de Docagem de Rotina e mais sete Ciclos Básicos. O Período de Atualização e Modernização visa a não obsolescência do submarino, por meio de substituições de subsistemas ou equipamentos atualizados ou acréscimo de capacidade ou facilidades não previstas no projeto original (GUIMARÃES, 1999).

O Ciclo de Atividades Final é formado por um "Período Operativo Final (POF)", com a duração de 72 meses: oito Ciclos Básicos seguido de um Período de Docagem de Rotina e mais sete Ciclos Básicos, mais um Período de Docagem Rápida e mais sete Ciclos Básicos. Após a última Missão de Patrulha Básica é iniciado o descomissionamento do submarino, com um Período de Docagem Final (PDF) (GUIMARÃES, 1999).

De acordo com o planejamento acima, são previstas 638 semanas de indisponibilidade para realização de rotinas de manutenção: 2 PAM, 2 PMG, 6 PDR e 82 PMR. A Figura 8 sintetiza o ciclo operacional de um SNA ao longo da vida útil.

Semanas

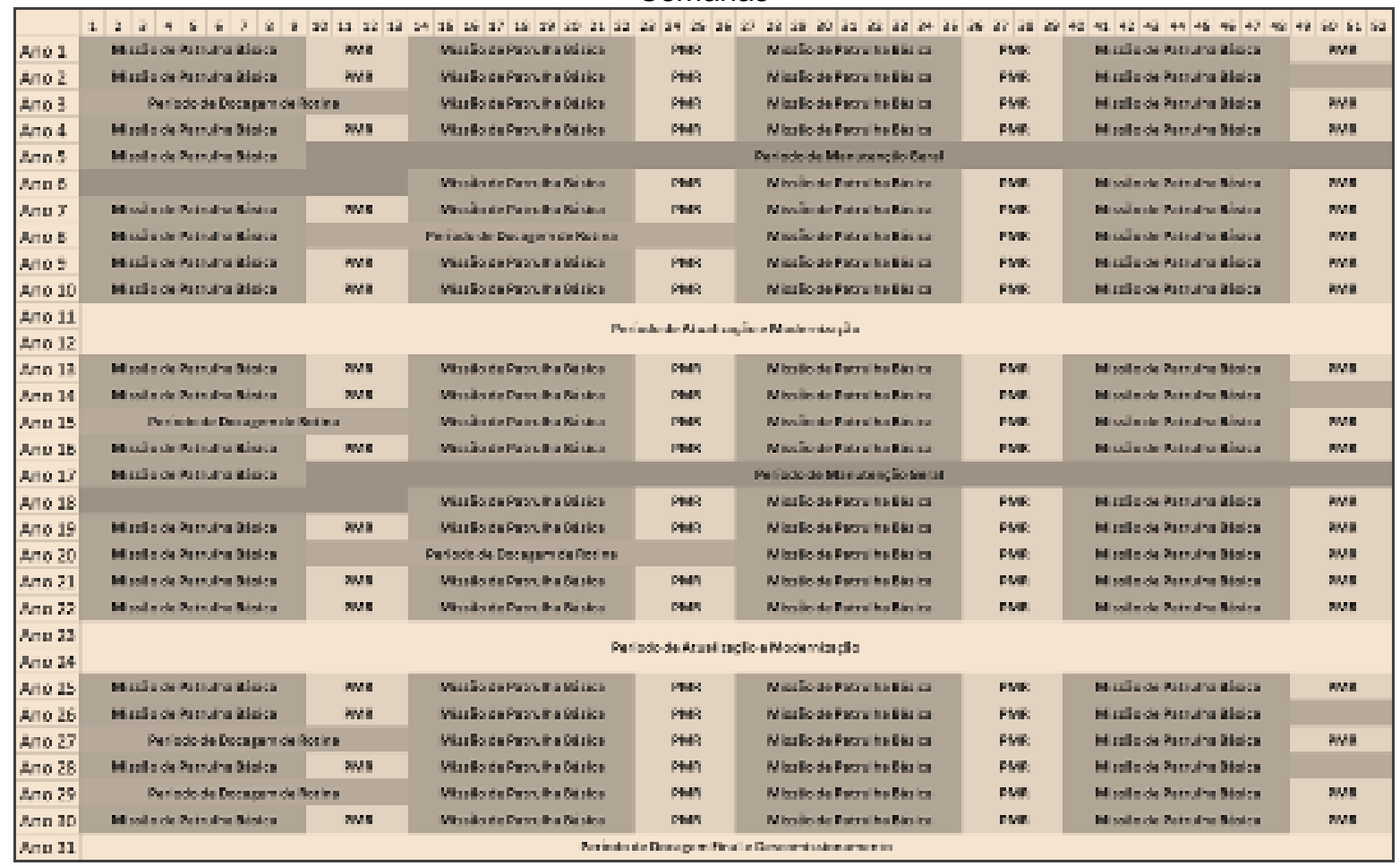

Figura 8 - Ciclo operacional de um Submarino Nuclear de Ataque ao longo da vida útil 
Considerando a vida útil de 1.560 semanas (30 anos), a disponibilidade técnica teórica para operação do submarino é de $59 \%$. Deve ser observado que estes cálculos são determinísticos e estão relacionados apenas ao sistema físico (hardware) do submarino. Na prática, as durações previstas para os ciclos de operações e os períodos de manutenção planejada deverão ser confirmadas como médias de distribuições probabilísticas expressas em temos de MTBF e MTTF durante o ciclo operativo do submarino, supondo sucesso do projeto no atendimento aos requisitos de confiabilidade.

Além do esforço gerencial para alcançar o nível de desempenho em disponibilidade técnica para atender o ciclo de operações pretendido, duas outras importantes dimensões devem ser tratadas de forma concomitante: a segurança e a capacidade de sobrevivência. Portanto ao longo do projeto devem ser definidos e analisados diversos cenários de operações atípicas e situações adversas.

Como se viu, o desempenho real do submarino em disponibilidade, segurança e capacidade de sobrevivência é uma variável probabilística resultante de uma dinâmica complexa de iterações entre sistemas físicos, softwares dos sistemas de supervisão e controle, fatores humanos inerentes à tripulação e aos elementos organizacionais de suporte. Portanto, dadas estas condições de contorno, o desdobramento dos requisitos de confiabilidade pretendidos desde o início do projeto até a sua conclusão, de forma a garantir níveis de desempenho e segurança satisfatórios ao "cliente", é uma atividade gerencial desafiadora que requer o emprego de ferramental analítico sofisticados.

\section{CONCLUSÃO}

Em face da sua complexidade e do potencial de acidentes catastróficos, o Submarino Nuclear é um sistema técnico-social classificado como uma Organização de Alta Confiabilidade (HRO), significando que esta organização deve responder satisfatoriamente ao conflito entre desempenho e segurança. Dado os altos riscos e custos envolvidos em um programa de desenvolvimento de submarinos nucleares, a decisão de construí-los só é justificada em face das expectativas dos benefícios proporcionados a um país em possuí-los. Esses benefícios só estarão ao alcance da sociedade por meio de uma capacidade efetiva de se projetar e construir um submarino, atendendo aos requisitos de desempenho em disponibilidade, segurança e sobrevivência. Com esse enfoque, o desempenho em confiabilidade torna-se fator crítico para o sucesso de um programa de desenvolvimento de submarinos nucleares.

Diferentemente de um item de baixa complexidade tecnológica, cuja confiabilidade pode ser alocada no projeto de engenharia, sendo possível fazer previsões de MTBF com razoável precisão, a confiabilidade de um sistema complexo, como o submarino nuclear, é o resultado de uma dinâmica de iterações entre sistemas físicos (hardware), softwares, sistema humano (tripulação) e elementos organizacionais. Dada tal complexidade, é necessário estabelecer um Programa de Confiabilidade para ser conduzido dentro da estrutura gerencial do projeto. 
O Projeto de Submarinos com o Foco em Confiabilidade deve trazer para o ambiente do projeto de engenharia considerações sobre incertezas e riscos. Portanto, além das melhores práticas em engenharia, gestão e qualidade, é vital para o sucesso do projeto de submarinos o uso competente de todo um ferramental analítico, incluído análises de probabilidade, técnicas de otimização e técnicas de simulação.

Em termos de confiabilidade, o desfio gerencial está no seu desdobramento, desde a fase de concepção do projeto do submarino, de forma a se obter o nível de desempenho requerido. Portanto, é vital a compreensão clara e precisa das suas funções, as condições operacionais e os ciclos operacionais e de manutenção.

A combinação do Apoio Logístico Integrado (ALI) e de uma estrutura de desenvolvimento guiado com etapas revisionais, cujas atividades são agrupada em fases e desenhadas de acordo com o estágio e o nível de detalhamento, se mostra adequada como estratégia para se garantir o desdobramento dos requisitos em confiabilidade ao longo do processo de desenvolvimento de submarinos nucleares.

\section{REFERÊNCIAS}

ASSOCIAÇÃO BRASILEIRA DE NORMAS TÉCNICAS. NBR 5462: Confiabilidade e Mantenabilidade. Rio de Janeiro: 1994.

BIERLY, P. E.; GALLAGHER, S.; SPENDER, J. C. Innovation and Learning in HighReliability Organizations: a case study of United States and Russian Nuclear Attack Submarines, 1970 - 2000. IEEE Transactions on Engineering Management, v. 55, n. 3 , august 2008.

BLANCHARD, B. S. Logistics Engineering and Management. 6th ed. New Jersey: Pearson Prentice Hall, 2004.

BLANCHARD, B. S.; FABRYCKY, W. J. System Engineering and Analysis. 4th ed. New Jersey: Pearson Prentice Hall, 2006.

BURCHER, R.; RYDILL, L. Concepts in Submarine Design. Cambridge: Cambridge University Press, 1994.

DAS, D.; PECHT, M. Design for Reliability. In: PECHT, M. Product Reliability, Maintainability and Suppotability Handbook. 2nd ed. Boca Raton: CRC Press Taylor \& Francis Group, 2009.

DIETER, G. E.; SCHMIDT, L. C. Engineering Design. 4th ed. New York: McGraw-Hill, 2008.

ESTADO MAIOR DA ARMADA. EMA-420: Normas para a Logística de Material. Marinha do Brasil: Brasília, 2002.

GUIMARÃES, L. S. Síntese de Doutrina de Segurança para Projeto e Operação de Submarinos Nucleares. 1999. 645 p. Tese (Doutorado) - Escola Politécnica, Universidade de São Paulo, São Paulo, 1999. 
HOLANDA, A. B. Novo Dicionário Aurélio da Língua Portuguesa. 3.ed. Rio de Janeiro: Ed. Positivo, 2004.

INTERNATIONAL ELECTROTECHNICAL COMMISSION. IEC 60300-1: Dependabiblity Management - Part 1: Dependability Management Systems. 2nd ed. Geneve: 2003.

KAMINSKI, P. C. Desenvolvendo Produtos com Planejamento, Criatividade e Qualidade. Rio de Janeiro: LTC, 2000.

KORMILITSIN, Y. N.; KHALIZEV, O. A. Theory of Submarine Design. SaintPetersburg: Saint-Petersburg State Maritime Technical University, 2001.

MURTHY, D. N. P.; RAUSAND, M.; VIRTANEN, S. Investment in New Product Reliability. Engineering and System Safety Journal, v. 94, 2009.

O'CONNOR, P. D. T. Pratical Reliability Engineering. 4th ed. West Sussex: John Wiley \& Sons Ltd, 2002.

SLACK, N.; CHAMBERS, S.; JOHNSTON, R. Administração da Produção. 2.ed. São Paulo: Ed. Atlas, 2008.

VASCONCELOS, M. C.; SANTOS, T. M. Projeto de Sistemas Oceânicos II: multipurpose ROV support vessel. Disponível em <http://www.oceanica.ufrj.br/deno/prod_academic/relatorios/2009/marceloC_thiagoM /relat1/>. Acessado em 30 maio 2011. 